\title{
The Novel Photoluminescence Powder Synthesized from Zinc Carbonate Nanoparticles Associated with Fluorescein Dye For Its Latent Fingerprint Detection
}

\author{
KONGSAK PATTARITH ${ }^{1}$ and RACHADAPORN BENCHAWATTANANON ${ }^{2 *}$ \\ 1'Department of Chemistry, Faculty of Science, Buriram Rajabhat University, Buriram 31000, Thailand. \\ ${ }^{2}$ Integrated Science, Forensic Science Division, Faculty of Science, Khon Kaen University, \\ Khon Kaen 40002, Thailand. \\ ${ }^{*}$ Corresponding author E-mail: rachadaporn@ @ku.ac.th \\ http://dx.doi.org/10.13005/ojc/360204
}

(Received: March 12, 2020; Accepted: April 13, 2020)

\begin{abstract}
Fingerprint verification in crime scene is a basic method, but it is very important thing to be linked to the offender. Using fluorescent powder in passive fingerprint authentication will increase the performance of fingerprint detection for an identification. In this study, the new fingerprint powder has been synthesized by solvothermal method using zinc carbonate and fluorescein dye as main precursor using ethanol as a solvent. The synthetic fluorescent powder is also inspected for trial features with various diagnostic tools. As follows, detecting a micro texture with scanning electron microscope (SEM), absorption with UV-Vis diffuse reflectance spectroscopy (DRS), examining the notation characteristics with X-ray diffraction (XRD) and UV light at wavelength $254 \mathrm{~nm}$ and 366 $\mathrm{nm}$. Testing of the synthetic fingerprint fluorescent powder is done by swiping on the surface with a latent fingerprint. For studying optimum conditions affecting the fingerprint detection, there are general following types of textures including glass, metal, plastic and ceramics, and the appearance of smooth, curve and rough surfaces. It is also studies on the effects of duration and temperature of the retention affecting the fingerprints which have been inspected by the trial. For the synthetic fluorescent powder, its inspection can be used on each surface of different types with a good surface appearance. It has a long retention period of more than 30 days at room temperature and more than 15 days at a temperature of $90^{\circ} \mathrm{C}$, and it can also be used to detect the fingerprints with a clear overlap. From this study, it is demonstrated that the synthetic fluorescent powder has a very excellent performance and has high possibility to be used to detect the passive fingerprints as an object witness in forensic processes.
\end{abstract}

Keyword: Photoluminescence, Latent fingerprint, Solvothermal method, Zinc carbonate, Fluorescein dye.

\section{INTRODUCTION}

Because of the unique and unchanging characteristics, fingerprints constitute one of the most crucial clues of physical evidence in forensic investigation, with latent fingerprints, the invisible

This is an Open Access article licensed under a Creative Commons license: Attribution 4.0 International (CC- BY). Published by Oriental Scientific Publishing Company @ 2018

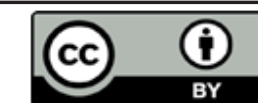


residue deposited by a person's hand when touching a surface, being the common form found at crime scene ${ }^{1}$. Among all biometric recognition methods, fingerprint recognition is considered one of the most technologically and powerful approaches for personal identification ${ }^{2}$.

For fingerprint detection, material surface is often a critical variant that often causes difficulties and can affect fingerprint detection, such as various kinds of paper, fiber, textile, leather, plastic, masking sheet, or human skin, etc. ${ }^{3}$. The fingerprint that appears on the surface is divided into three types: three-dimensional (plastic print) fingerprint visible with the naked eye (visible print) and a passive fingerprint (latent print). For 3-dimension fingerprints, there are three-dimensional morphology, which is the result of a semi-solid material surface, such as wax clay, etc. The fingerprint visible to the naked eye is the fingerprint that appears. The passive fingerprint is invisible or unclear fingerprint. Therefore, it is necessary to have any process to cause the image to be taken ${ }^{4}$. Analyzed techniques that help multi-finger passive checks may include the physical, chemical, and light techniques either or everything is assembled. The evidence means it can be captured by law, and what can be presented in the court to prove the fact of the case. Forensic evidence is reliable and acceptable in court, can be brought into the process of consideration in order to prove the actual occurrence of the case and can lead to criminal penalties in accordance with law. When a crime case occurs. Most of the things found in the scene are fingerprints. The palms of the feet are one of the branches in the Human identification. Because the gesture appears on your finger. The palm of the hands and the feet of the man have a special characteristic of the stripes that appear on the fingers. Different palms and feet. Forensic research studies related to covert fingerprint detection with fluorescent chemicals in conjunction with multiple light generators, the frequency is also very minimal. Development of proven capabilities the fingerprint side is also very necessary to develop further. Therefore, it is the source of the need to study new materials. It is used to detect the passive fingerprints on various materials to enhance and develop new techniques to detect and analyze passive fingerprints more efficiently and reliably.
In recent years, nanoparticles exhibit a variety of potential applications in several industries due to its excellent chemical, physical, optical, mechanical, electrical and magnetic properties ${ }^{5}$.

In this study, we fabricate novel photoluminescent nano-power based on $\mathrm{ZnCO}_{3}$ associated with fluorescein dye for the detection of latent fingerprints. Influential factors that affect the monitored passive fingerprints were also examined, including temperature and time to maintain a passive fingerprint that has been validated, as well as passive fingerprint verification with overlapping fingerprints, synthetic materials can be used to validate fingerprint verification to prove a personal identity in forensic work.

\section{MATERIALS AND METHODS}

\section{Chemicals and characterization}

Zinc carbonate $\left(\mathrm{ZnCO}_{3}\right)$, Fluorescein $\left(\mathrm{C}_{20} \mathrm{H}_{12} \mathrm{O}_{5}\right)$, Absolute ethanol were purchase from Sigma-Aldrich Chemical Co. Ltd. All the chemicals were used without any further purification. Morphological analysis of prepared Photoluminescence powder was carried out by using Scanning Electron Microscope (LEO/1450). The phase purity and crystal structure were examined by using a X-ray diffractometer (Bruker/D8 Advance). Ultraviolet visible (UV) absorption spectra measurements were carried out on UV-Vis diffuse reflectance spectrometer (SHIMADZU/UV-1900). The latent fingerprint images were photographed by using a Smartphone camera (Oppo A57).

\section{Preparation of photoluminescence powder}

The synthesis of fluorescent dust from zinc carbonate $\left(\mathrm{ZnCO}_{3}\right)$ by means of dispersion in the Hellevoetsluis-A-Victor (solvothermal reactor) is prepared by 2 and $0.002 \mathrm{~g}$ of fluorescent and fluorescence zinc, respectively. The dispersion in ethanol $50 \mathrm{~mL}$ then reacts in solvothermal reactor at a temperature of $150^{\circ} \mathrm{C}$ for $3 \mathrm{~h}$ after it is left to cool. At room temperature Filter, remove sludge and remove sludge, drying with pressure-reducing evaporation. Store it in a humidifier and don't get exposed to light.

\section{Characterization of synthetic photoluminescence powder}

The synthesis of fluorescent powder from zinc carbonate has been analyzed to detect the 
characteristic of the microstructure and particle characteristics with scanning electron microscope (SEM) techniques. UV-Vis diffuse reflectance spectroscopy (DRS) To measure the absorption of the powder fluorescent. It is done to inspect the crystalline characteristics of crystals with $\mathrm{X}$-ray diffraction (XRD) technique and UV light at wavelengths of $254 \mathrm{~nm}$ and $366 \mathrm{~nm}$.

\section{Latent fingerprint detection test}

This research has studied the removal of fluorescent powder from the synthesis of a test. check for passive fingerprints by means of powder extraction on the fingerprint footprint in various conditions, such as detecting fingerprints on the surface of different materials. Such as metal sheets glass, ceramics and plastic, both with curved and rugged surface and passive fingerprint detection with overlap finger overlap. It also studies the effect of temperature and retention time of therefore-branded fingerprints. A passive fingerprint check is made by comparing light stories from photos under ultraviolet light at wavelength 254 and $366 \mathrm{~nm}$.

\section{RESULTS AND DISCUSSION}

\section{Zinc carbonate and fluorescein photolum- inescence powder}

The effect of the synthesis of fluorescent powder is the key factor that affects the illumination of synthetic materials which the solvent and the amount of time it takes to synthesize by the experimental results shown in Table 1. From Table 1 , the experimental result suggests that the solvent is suitable for synthesis, the fluorescent dust is ethanol and takes $3 \mathrm{~h}$ of reaction at a temperature of $150^{\circ} \mathrm{C}$, which will illumination under the ultraviolet light, both at short waves $(254 \mathrm{~nm})$ and waves (366 nm). Since the fluorescence can be dissolved in ethanol solvent and can be dispersed into zinc carbonate better than using water as a solvent, since the molecules of the water are going to surround the particles of zinc carbonate, the fluorescence cannot be propagated into the particles of zinc carbonate. In the event that the reaction time is too long ( $6 \mathrm{~h}$ ), the fluorography is degraded because it is in a hot and high pressure sector.

Table 1: The effects of solvent and time on the synthesis of fluorescent powder

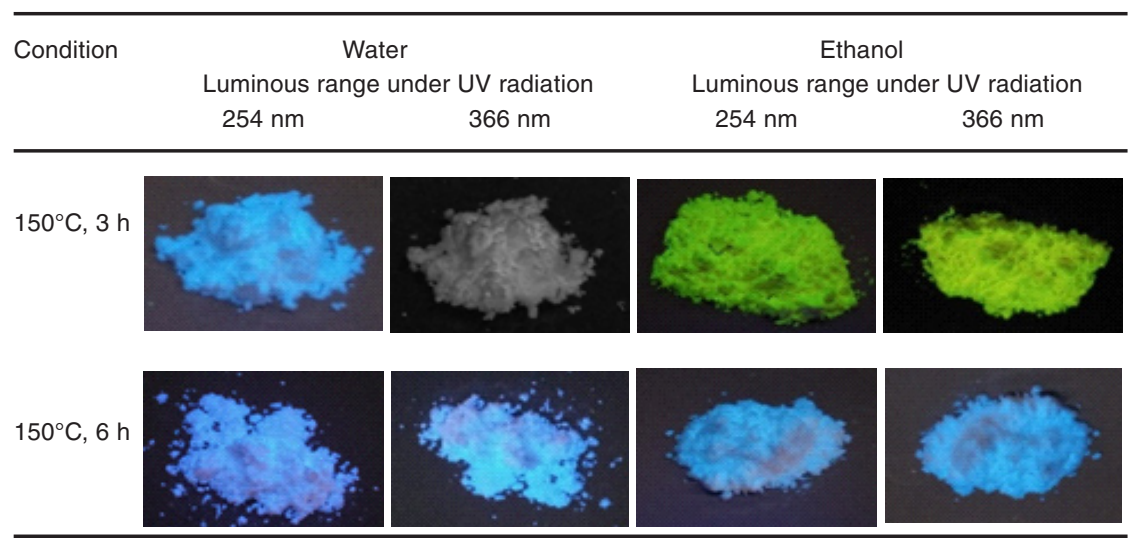

UV-Visible diffuse reflectance spectroscopy (DRS)

The result is a study of pure carbonate zinc in contrast to synthetic powder (Fig. 1). Find that the absorption value of two types of substances has a different absorption value. By pure carbonate zinc $(A)$ There is a light absorption value in the wavelength range below $200 \mathrm{~nm}$. While synthetic powder has a value of ultraviolet light absorption in the wavelength range of 200-400 $\mathrm{nm}$ and absorbs light during a visible light range at wavelength $500 \mathrm{~nm}$. This corresponds to the physical characteristics of the synthetic compound that is orange and from which the synthetic substances absorb ultraviolet light in a wide wavelength range, which is a characteristic of fluorescent. The energy that is absorbed is to stimulate electrons from the ground state to the stimulus state. When the electrons return to the ground state, it emits energy in the form of light. This causes the illumination condition to occur. 


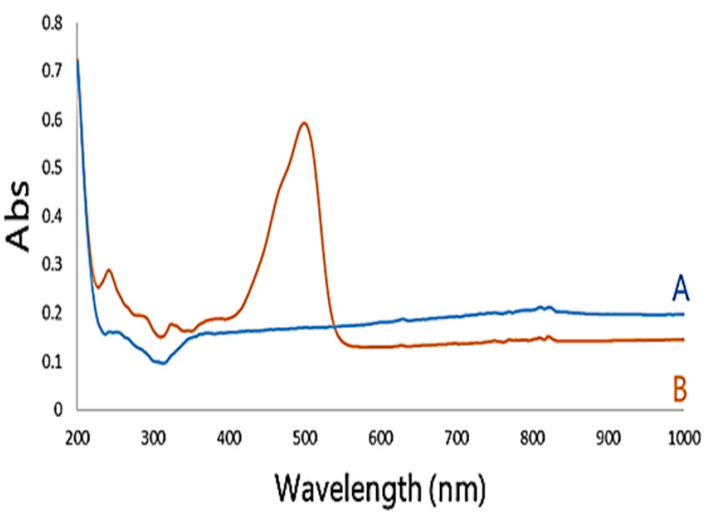

Fig. 1. UV-Vis diffuse reflectance spectra of pure zinc carbonate (A) and artificial fluorescent powder (B)

\section{Scanning electron microscope (SEM)}

The results study the surface appearance, microstructure and appearance of particles of synthetic fluorescent powder, compared with pure carbonate zinc in solid form (Fig. 2). In the study of the morphology, the appearance of pure zinc carbonate particles (Fig. $2 \mathrm{~A}$ and B) particles are being into large particles due to the toughness of sticky bonding between the particles of zinc carbonate. When the zinc carbonate is processed, the particles have a smaller AVR and separated from each other (Fig. $2 \mathrm{C}$ and D), as the molecules of the fluorescent are inserted in accordance with the particles of zinc carbonate. This allows the particle of zinc carbonate to be more fragmented.

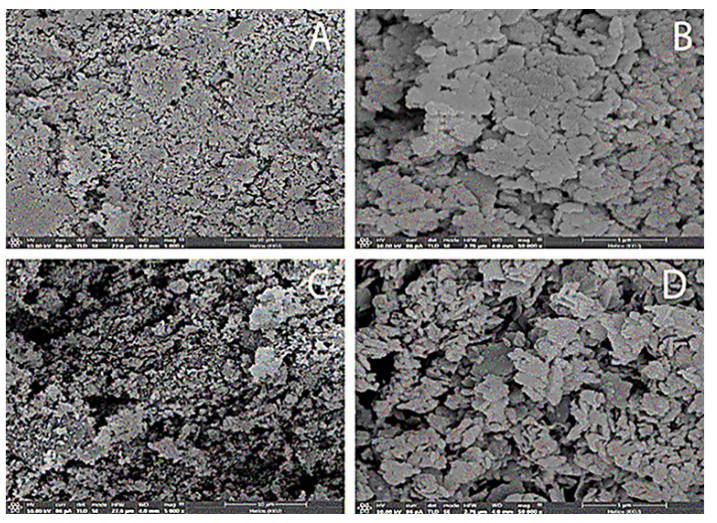

Fig. 2. SEM images of zinc carbonate (A: 5,000x, B: 50,000x) and synthetic powder (C: 5,000x, D: 50,000x)

\section{X-ray diffraction (XRD)}

Visual notation of synthetic powder crystal, comparing the zinc carbonate with diffraction technique (X-ray diffractometer: XRD) Displays XRD pattern, as shown in Fig. 3. From Fig. 3, the XRD pattern of synthetic dust that is from $\mathrm{ZnCO}_{3}$ reaction to Fluorescein in Fig. $3 \mathrm{~B}$ has a pattern matching the XRD pattern of pure carbonate zinc crystals (Fig. $3 \mathrm{~A}$ ), which are used as precursor. It shows that the powder is synthesized by the solvothermal method, and is still characterized by the fundamental notation of zinc carbonate crystals. No changes have been made.

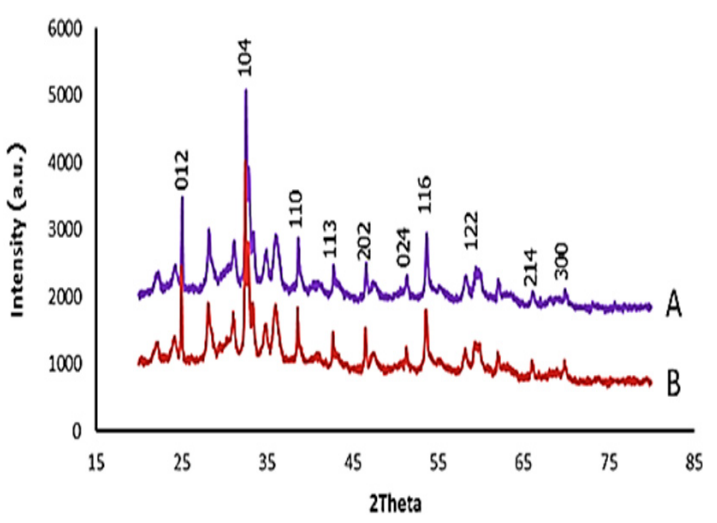

Fig. 3. XRD patterns of zinc carbonate (A) and the synthetic powder (B)

\section{Latent fingerprint authentication with fluorescent powder}

From the examination of the latent fingerprint on the surface of various types of materials with different surface characteristics and take pictures under ultraviolet light to compare the sharpness of latent fingerprints on various materials. The results are shown in Table 2. From Table 2, results of the use of synthetic fluorescent powder to check the hidden fingerprint on the surface of various materials It was found that fluorescent powder can adhere to passive fingerprint well and can be inspected by providing fluorescent powder under ultraviolet light as for the sharpness of the fingerprint, it depends on the surface characteristics such as flatness, roughness and surface scratches. Since the surface of some materials is a rough surface when touched by a finger any area with a smooth surface, the fat from the fingers will stick directly to the material, while the rough areas on the skin of the fingers will not stick very well ${ }^{6}$. Resulting when swiping for hidden fingerprints in the area where the fat from Hands can absorb a lot of synthetic dust. Areas with a rough surface can absorb only a small amount of synthetic dust. As a result, the fingerprint that has been detected has reduced sharpness in some areas. In addition, it was found that the glow of the dust will glow more than when under ultraviolet light at the wavelength of $254 \mathrm{~nm}$. 
Table 2: Fingerprint identification with fluorescent dust on various types of materials

\begin{tabular}{llc}
\hline Material & $\begin{array}{c}\text { Photo sharpness of passive fingerprint } \\
\text { Luminous range under UV radiation } \\
254 \mathrm{~nm}\end{array}$ \\
\hline Brass & $366 \mathrm{~nm}$ \\
\hline Stainless steel & & \\
\hline
\end{tabular}

Effect of time on storing latent fingerprints examined with the fluorescent powder

Test for the persistence of latent fingerprints with mirrors, which are inspected by fluorescent powder in different time periods. By comparing with the initial latent fingerprint identification The test results are shown in Table 3. From Table 3, it is found that the storage time will affect the fluorescent ability of synthetic powder. When the storage period has elapsed, the fluorescence of synthetic materials is reduced. Both under ultraviolet light at wavelengths 254 and $366 \mathrm{~nm}$, however, when storing fingerprints for 32 days, although the fluorescence is reduced, but still can see details of the hidden fingerprint clearly

Table 3: Effect of time on the clarity of latent fingerprint storage

\begin{tabular}{llll}
\hline Storage period & \multicolumn{2}{c}{$\begin{array}{c}\text { Photo of latent fingerprint storage time } \\
\text { Visible light }\end{array}$} \\
\hline Starting day & $\begin{array}{c}254 \mathrm{~nm} \\
366 \mathrm{~nm}\end{array}$ \\
\hline After 17 days & & \\
\hline After 32 days & \\
\hline
\end{tabular}


Effect of temperature on storage of latent fingerprints examined with fluorescent powder

Study for the optimum temperature for storing latent fingerprints that are brushed with fluorescent material by storing it under different temperatures for 16 days. The results are shown in Table 4. From Table 4, the study of the effect of temperature on the fluorescence of synthetic powder, studying for
16 days at room temperature $60^{\circ} \mathrm{C}$ and $90^{\circ} \mathrm{C}$. It was found that the temperature that has studied affects the fluorescence of synthetic powder because at $90^{\circ} \mathrm{C}$ in the storage period of day ${ }^{7}$, the powder Synthetic began to less illumination which is caused by fluorescent fluorescence, which decays when exposed to high heat for a long period of time. Therefore, to collect latent fingerprints should not do at high temperatures.

Table 4: Effect of temperature on latent fingerprint storage under UV-light at $254 \mathrm{~nm}$

\begin{tabular}{llll}
\hline Storage duration & \multicolumn{2}{c}{ Storage temperature } \\
& Room temperature & $60^{\circ} \mathrm{C}$ & $90^{\circ} \mathrm{C}$ \\
\hline & & & \\
\hline & & & \\
\hline
\end{tabular}

The latent fingerprint images when overlapped with synthetic materials

The study of the effects of latent fingerprint overlays is an important factor for detecting latent fingerprint. Since it cannot be determined that the desired passive fingerprint needs to be free from other passive fingerprints, the overlapping latent fingerprints test is one of the important factors in the synthesis of fluorescent powder ${ }^{7}$. The results of the study of overlapping fingerprints are shown in Table 5. From Table 5, the study of the effects of latent fingerprint overlap on the fluorescence of synthetic powder examined by ultraviolet light at the wavelength of $254 \mathrm{~nm}$ and $366 \mathrm{~nm}$, found that the fluorescence of synthetic powder can clearly show the pattern of latent fingerprints on both fingerprints. Can be synthesized, therefore it is a suitable alternative to detecting overlapping fingerprints.
Table 5 The latent fingerprint images when superimposed with fluorescent powder

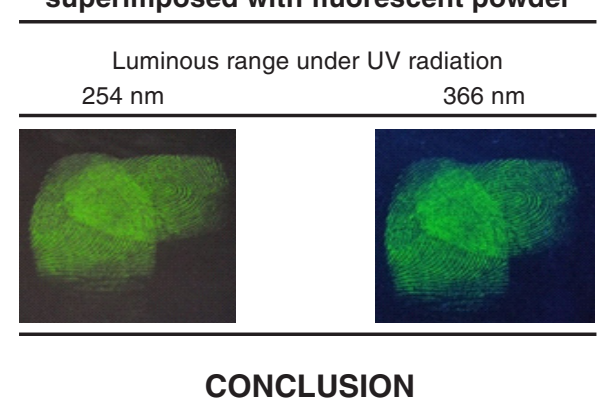

From the study of fluorescent powder synthesis for forensic fingerprint test from zinc carbonate, reacting with fluorescence in solvothermal reactivity at $150^{\circ} \mathrm{C}$ for $3 \mathrm{~h}$ in ethanol solvent has the best fluorescence properties of synthetic substances, both at 254 and $366 \mathrm{~nm}$ wavelengths, and from the study of factors affecting the rate of fluorescence of synthetic dust, such as type of material, finger overlay, duration and temperature of fingerprints 
storage Found that the type of material And the overlay result of the hidden fingerprint It does not affect the clarity and glow rate of synthetic dust, while the storage time and temperature will affect the fluorescence of synthetic materials. Since the properties of phosphor when exposed to high heat or stored for a long time, it will cause the substance to decompose.

\section{ACKNOWLEDGMENT}

This work was supported by Faculty of Science, Khon Kaen University, Khon Kaen, Thailand.

\section{Conflict of interest}

The authors declare no conflict of interest.

\section{REFERENCES}

1. Alsolmy, E.; Abdelwahab, W.M.; Martinez, V.; Henary, M.; Patonay, G. J. Photochem. Photobiol. A Chem., 2020, 392, 112416.

2. Ghubish, Z.; Saif, M.; Hafez, H.; Mahmoud, H.; Kamal, R.; El-Kemary, M. J. Molec. Struct., 2020, 1207, 127840.

3. Champod, C.; Lennard, C.J.; Margot, P.; Stoilovic, M. Fingerprints and Other Ridge Skin Impressions, $2^{\text {nd }}$ Edition., 2016, 1-428.

4. Seah, L.K.; Dinish, U.S.; Phang, W.F.; Chao,
Z.X.; Murukeshan, V.M. Forensic Sci. Int., 2005, 152(2), 249-257.

5. Ashwini, S.; Prashantha, S.C.; Naik, R.; Naik, Y.V.; Nagabhushana, H.; Jnaneshwara, D.M. Optik., 2019, 192, 162956.

6. Jasuja, O.P.; Singh, G.D.; Sodhi, G.S. Sci. Justice., 2008, 48(3), 141-145.

7. Sobral, G.A.; Gomes, M.A.; Macedo, Z.S.; Alencar, M.A.R.C.; Novais, S.M.V. Bull. Mater. Sci., 2016, 39(6), 1565-1568. 